\title{
Decreased Proteasomal Activity Causes Age-Related Phenotypes and Promotes the Development of Metabolic Abnormalities
}

\author{
Utano Tomaru, ${ }^{*}$ Satomi Takahashi, ${ }^{*}$ \\ Akihiro Ishizu, ${ }^{\dagger}$ Yukiko Miyatake, ${ }^{*}$ Aya Gohda, ${ }^{*}$ \\ Sayuri Suzuki, ${ }^{*}$ Ayako Ono, ${ }^{*}$ Jiro Ohara, ${ }^{*}$ \\ Tomohisa Baba, ${ }^{\ddagger}$ Shigeo Murata, ${ }^{\S}$ Keiji Tanaka, \\ and Masanori Kasahara*

\begin{abstract}
From the Department of Pathology, * Hokkaido University Graduate School of Medicine, Sapporo; the Faculty of Health Sciences, ${ }^{\dagger}$ Hokkaido University, Sapporo; the Division of University, Kanazawa; the Laboratory of Protein Metabolism, Graduate School of Pharmaceutical Science, The University of Tokyo, Tokyo; and the Laboratory of Protein Metabolism, "T Tokyo Metropolitan Institute of Medical Science, Tokyo, Japan
\end{abstract} \\ Molecular Bioregulation, ${ }^{\ddagger}$ Cancer Research Institute, Kanazawa
}

The proteasome is a multicatalytic enzyme complex responsible for the degradation of both normal and damaged proteins. An age-related decline in proteasomal activity has been implicated in various agerelated pathologies. The relevance of decreased proteasomal activity to aging and age-related diseases remains unclear, however, because suitable animal models are not available. In the present study, we established a transgenic (Tg) mouse model with decreased proteasomal chymotrypsinlike activity. Tg mice exhibited a shortened life span and developed age-related phenotypes. In $\mathrm{Tg}$ mice, polyubiquitinated and oxidized proteins accumulated, and the expression levels of cellular proteins such as Bcl-xL and RNase $L$ were altered. When Tg mice were fed a high-fat diet, they developed more pronounced obesity and hepatic steatosis than did wild-type mice. Consistent with its role in lipid droplet formation, the expression of adipose differentiation-related protein (ADRP) was elevated in the livers of $\mathrm{Tg}$ mice. Of note, obesity and hepatic steatosis induced by a high-fat diet were more pronounced in aged than in young wild-type mice, and aged wild-type mice had elevated levels of ADRP, suggesting that the metabolic abnormalities present in $\mathbf{T g}$ mice mimic those in aged mice. Our results provide the first in vivo evidence that decreased proteasomal chymotrypsin-like activity affects longevity and aggravates age-related metabolic disorders, such as obesity and hepatic steatosis. (Am J Pathol 2012, 180:963-972; DOI: 10.1016/j.ajpath.2011.11.012)

Protein degradation is a major intracellular function, responsible not only for cellular housekeeping but also for regulation of important cellular functions. Two major proteolytic systems are responsible for most intracellular protein turnover: the lysosomal system and the ubiquitin (Ub) proteasome system. ${ }^{1}$ The latter contributes to the maintenance of cellular homeostasis and protein quality control, and functions as a regulator of many essential cellular processes, including proliferation, cell cycling, gene transcription, apoptosis, antioxidant responses, and immune reactions. ${ }^{2,3}$ Proteasomes are multisubunit complexes made up of a core particle, known as the $20 \mathrm{~S}$ proteasome, and additional subunits comprising the 195 complex. Proteolysis is conducted by three $\beta$ subunits, $\beta 1, \beta 2$, and $\beta 5$, which have caspase-like, trypsin-like, and chymotrypsin-like activities, respectively. ${ }^{4}$ It has been assumed that the relative importance of the three proteolytic activities varies across substrates and depends on the proteins being degraded. ${ }^{5}$ However, little is known about the importance of each proteasomal activity in vivo.

Recently, the proteasome has emerged as an attractive target for cancer therapy, because proteasome inhibition has produced encouraging antitumor activity in preclinical studies and clinical trials. Bortezomib (originally codenamed PS-341), the first proteasome inhibitor tested in clinical trials, has been used for treating multiple myeloma patients. ${ }^{6}$ This drug has also been shown to be effective with manageable toxicity for mantle cell and other lymphomas, leukemias, and solid malignancies, in-

Supported in part by grants-in-aid for scientific research from the Ministry of Education, Culture, Sports, Science and Technology of Japan (U.T.).

Accepted for publication November 4, 2011

Supplemental material for this article can be found at http://ajp. amjpathol.org or at doi: 10.1016/j.ajpath.2011.11.012.

Address reprint requests to Utano Tomaru, M.D., Ph.D., Kita-15, Nishi-7, Kita-ku, Sapporo 060-8638, Japan. E-mail: tomaruu@med.hokudai.ac.jp. 
cluding non-small cell lung carcinoma. ${ }^{6}$ Although different factors regulate apoptosis in individual carcinoma cell models, it is plausible that bortezomib promotes apoptosis by shifting the balance from antiapoptotic signals toward proapoptotic signals through modulation of the translocation of nuclear factor- $\kappa \mathrm{B}(\mathrm{NF}-\kappa \mathrm{B}){ }^{7}$

Physiologically, proteasomal activity decreases with age. ${ }^{8-11}$ Because the proteasome plays an essential role in cellular processes, an age-associated decline in proteasome function is assumed to contribute to the development of age-related pathology and to the aging process itself. ${ }^{10}$ Indeed, recent evidence indicates that mouse hepatocytes with impaired assembly of 205 proteasomes undergo premature senescence. ${ }^{12}$ Furthermore, reduced 26S proteasome assembly in Drosophila was shown to shorten life span and enhance the development of neurodegenerative phenotypes. ${ }^{11}$ Nonetheless, there is no direct evidence linking decreased $20 S$ proteasomal activity and age-related pathology in mammals, because in mice deficiency in catalytic subunits $\beta 1, \beta 2$, or $\beta 5$ is lethal, and no other suitable animal models are available.

In the present study, we generated transgenic $(\mathrm{Tg})$ mice with decreased proteasomal chymotrypsin-like activity by introducing mouse $\beta 5 \mathrm{t}$ as a transgene. $\beta 5 \mathrm{t}$ is a recently discovered $\beta 5$-like subunit of the $20 \mathrm{~S}$ proteasome expressed exclusively in the thymus. ${ }^{13}$ In the thymic cortex, $\beta 5$ t is incorporated into 205 proteasomes in place of $\beta 5$ or $\beta 5 i$ and forms a specialized type of proteasomes, known as thymoproteasomes, that seem to be important for positive selection of T cells. ${ }^{13-15}$ Because $\beta 5 t$ has only weak chymotrypsin-like activity and seems to be preferentially incorporated into $20 \mathrm{~S}$ proteasomes over $\beta 5$ or $\beta 5 \mathrm{i},{ }^{16}$ we reasoned that forced expression of this subunit in vivo would generate an animal model with decreased proteasomal chymotrypsin-like activity. We show here that the reduction of proteasomal chymotrypsin-like activity in vivo results in a shortened life span and the development of age-related phenotypes. We also show that mice with decreased proteasomal chymotrypsin-like activity develop more profound obesity and hepatic steatosis than wild-type (WT) mice when challenged with a high-fat diet (HFD).

\section{Materials and Methods}

\section{Generation of Tg Mice and Animal Experiments}

Mouse $\beta 5$ t cDNA was ligated to the CAGGS vector, ${ }^{17}$ which allows for ubiquitous expression of a transgene under the control of the cytomegalovirus immediate-early enhancer and the chicken-actin promoter/enhancer. The expression construct was injected into C57BL/6 fertilized ova; integration of the $\beta 5 \mathrm{t}$ transgene was detected in three of the pups born from the DNA-injected ova. The transgene was transmitted into the offspring in two independently derived transgenic lines. Both transgenic lines showed similar phenotypes, with low weight and a short life span, compared with WT littermates. Both Tg and WT mice were housed on a 12-hour light-dark cycle in climate-controlled, pathogen-free barrier facilities. For feed- ing experiments, 6-week-old Tg and WT male mice were fed a HFD or a normal control diet (CD) (HFD-60 or AIN-93G; Oriental Yeast, Tokyo, Japan) and water ad libitum for 16 weeks. For aged mice, we used 76-weekold WT mice; they were fed HFD or CD for 16 weeks. All animal experiments were conducted according to the Guidelines for the Care and Use of Laboratory Animals at Hokkaido University Graduate School of Medicine.

\section{Cell Isolation and Cell Culture}

Primary fibroblastic cells (FCs) and hepatocytes were isolated from Tg and WT male mice using collagenase digestion. FCs were grown in Dulbecco's modified Eagle's medium supplemented with $20 \%$ fetal bovine serum, $100 \mathrm{U} / \mathrm{mL}$ penicillin, and $100 \mathrm{U} / \mathrm{mL}$ streptomycin in an atmosphere of $5 \% \mathrm{CO}_{2}$ and $95 \% \mathrm{O}_{2}$ at $37^{\circ} \mathrm{C}$. FCs were assayed for proteasomal activity during passages 3 to 4; primary hepatocytes were assayed immediately after isolation. FCs from WT mice were treated with 5 to $50 \mathrm{nmol} / \mathrm{L}$ of bortezomib (Janssen Pharmaceuticals, Tokyo, Japan) for 20 hours and subjected to Western blot analysis. Hepatocytes from WT mice were incubated in oleic acid-containing medium (600 $\mu \mathrm{mol} / \mathrm{L}$ ) with or without $100 \mathrm{nmol} / \mathrm{L}$ bortezomib for 48 hours and then were subjected to staining with Oil Red O (SigmaAldrich, St. Louis, MO) and Western blot analysis.

\section{Histological Analysis}

Formalin-fixed tissue sections were stained with H\&E. The thickness of the dermal adipose and muscle layers was quantified by random measurements of the length of individual skin samples. To estimate lipid accumulation, frozen liver sections or isolated primary hepatocytes were stained with Oil Red O solution. For immunohistochemical detection of $\mathrm{Ub}$, tissue slides were processed for antigen retrieval by a standard microwave heating technique, and incubated with anti-Ub antibody (Ab) (DakoCytomation Japan, Tokyo, Japan), followed by detection with streptavidinbiotin-horseradish peroxidase (DakoCytomation Japan). To detect aggresomes, FCs were cultured in chamber slides, and slides were stained according to the manufacturer's instructions (ProteoStat aggresome detection kit; Enzo Life Sciences, Farmingdale, NY; Lausen, Switzerland). For immunofluorescence staining for adipose differentiation-related protein (ADRP), hepatocytes on chamber slides were washed twice in PBS, fixed with $4 \%$ paraformaldehyde for 30 minutes, and permeabilized with $0.1 \%$ Triton-X surfactant in PBS for 5 minutes. After a washing, the slides were incubated in PBS with 10\% goat serum for 1 hour at room temperature, followed by overnight incubation with anti-ADRP Ab (Abcam Japan, Tokyo, Japan) at $4^{\circ} \mathrm{C}$. Sections were stained with BODIPY 493/503 (Invitrogen, Tokyo, Japan) to detect intracellular lipid, and labeled with Alexa Fluor 594-conjugated goat antichicken IgG (Invitrogen) and Hoechst 33342 dye (Enzo Life Sciences) for nuclear staining. 


\section{Immunoprecipitation and Western Blot Analysis}

Tissues were lysed in a buffer containing $150 \mathrm{mmol} / \mathrm{L}$ $\mathrm{NaCl}, 20 \mathrm{mmol} / \mathrm{L}$ Tris-HCl (pH 7.5), 0.2\% NP-40 detergent, and $1 \mathrm{mmol} / \mathrm{L}$ dithiothreitol, and centrifuged at $15,000 \times g$ for 10 minutes. The supernatants $(10 \mu \mathrm{g}$ aliquot of total proteins) were subjected to SDS-PAGE and blotted onto nitrocellulose membranes. The blots were probed with Abs, and reacted with horseradish peroxidase-conjugated anti-rabbit IgG (Jackson ImmunoResearch, West Grove, PA) for immunodetection. The immune complexes were visualized by enhanced chemiluminescence (Amersham; GE Healthcare, Piscataway, $\mathrm{NJ}$ ) and analyzed by Image Gauge software version 2.1 (Fujifilm, Tokyo, Japan). For immunoprecipitation, tissue lysates (100 $\mu \mathrm{g}$ of protein) were incubated in a total volume of $150 \mu \mathrm{L}$ of $25 \mathrm{mmol} / \mathrm{L}$ Tris- $\mathrm{HCl}$ buffer $(\mathrm{pH} 7.5)$ containing $1 \mathrm{mmol} / \mathrm{L}$ dithiothreitol at $4^{\circ} \mathrm{C}$ for 2 hours with Abs bound to protein A Sepharose (Amersham). The beads were washed and boiled in SDS sample buffer. After centrifugation, supernatants were used for Western blot analysis. Each lane was loaded with $15 \%$ of the total amount of proteasomes precipitated with Abs. The Abs for $\beta 5, \beta 5 i, \beta 5 t$, and $\alpha 6$ were as described previously. ${ }^{13}$ The Abs for RNase $L$ were purchased from Santa Cruz Biotechnology (Santa Cruz, CA); the Abs for $\mathrm{Bcl}-\mathrm{xL}$ were purchased from Sigma-Aldrich. The Abs for cell death-inducing DNA fragmentation factor-45like effector protein (CIDE) A, CIDE B, fat-specific protein 27 (Fsp 27), and perilipin A were purchased from Abcam Japan. The Ab for Ub was purchased from Enzo Life Sciences. These antibodies were used according to the manufacturers' recommendations. All information, including the dilution and organism, may be provided via data sheets form the companies.

\section{Measurement of Proteasomal Activity}

FCs and hepatocytes were harvested into 96-well plates at 10,000 cells per well and were analyzed using proteasome activity assay kits (Proteasome-Glo cell-based assay; Promega, Madison, WI), according to the manufacturer's instructions. Briefly, cells were incubated with specific luminogenic proteasome substrates (Suc-LLVY-aminoluciferin for chymotrypsin-like activity, Z-LRR-aminoluciferin for trypsin-like activity, and Z-nLPnLD-aminoluciferin for caspase-like activity), and substrate luminescence was measured by a luminometer. The number of viable cells in each well was measured by ATP measurement methods using luciferase reactions (CellTiterGlo luminescent cell viability assay; Promega). All data were corrected by the number of viable cells and expressed as a substrate luminescence/ATP luminescence ratio. All measurements were made in triplicate.

\section{Cellular Senescence Assay}

To detect senescence-associated $\beta$-galactosidase, FCs in subconfluent cultures were stained using a cellular senescence assay kit (Cell Biolabs, San Diego, CA). Briefly, cells were washed with PBS, fixed for 5 minutes with fixing solution, and washed again with PBS. Cells were incubated overnight at $37^{\circ} \mathrm{C}$ in a $\mathrm{CO}_{2}$-free atmosphere with senes- cence-associated $\beta$-galactosidase staining solution. Positive cells were scored under light microscopy.

\section{Laboratory Data and Measurement of Triglycerides and Cholesterol}

Tail blood was collected and centrifuged to obtain clear sera. Blood total protein, albumin, aspartate transaminase, lactate dehydrogenase, and cholesterol levels were measured at the Kishimoto Clinical Laboratory (Sapporo, Japan). Serum leptin and insulin levels were determined via enzyme-linked immunosorbent assay, according to the manufacturer's instructions, at the Morinaga Institute of Biological Science (Tokyo, Japan). For glucose tolerance tests, mice fed the CD or a HFD for 12 weeks were fasted for 12 hours with full access to water. Fasted mice were administered D-glucose solution intraperitoneally at $1 \mathrm{~g} / \mathrm{kg}$ body weight. At 0, 30,60, or 120 minutes after administration, blood glucose levels were measured using a measuring device (OneTouch UltraVue; Johnson \& Johnson, Tokyo, Japan). Triglycerides and cholesterol in the liver tissue were measured at Skylight Biotech (Tokyo, Japan).

\section{Protein Assays}

Muscles were lysed in a buffer containing $150 \mathrm{mmol} / \mathrm{L}$ $\mathrm{NaCl}, 20 \mathrm{mmol} / \mathrm{L}$ Tris- $\mathrm{HCl}$ ( $\mathrm{pH} 7.5), 0.2 \% \mathrm{NP}-40$, and 1
A

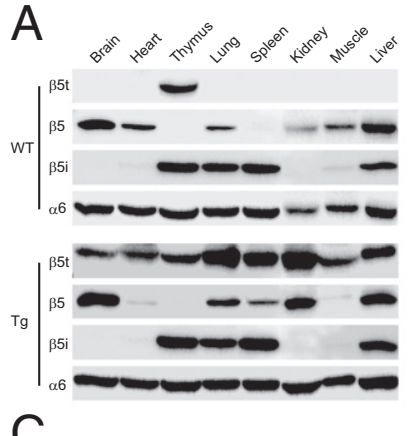

C

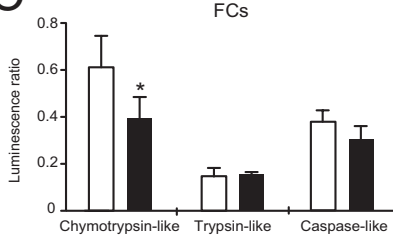

B

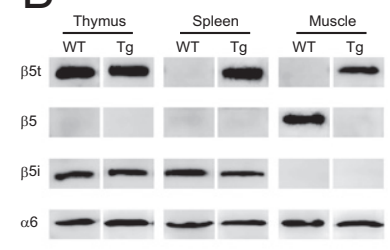

Figure 1. $\beta 5 \mathrm{t}$ is ubiquitously expressed and incorporated into $20 \mathrm{~S}$ proteasomes in Tg mice. A: Expression of $\beta 5 \mathrm{t}$ in WT and Tg mice. Tissue extracts were immunoblotted with $\beta 5 \mathrm{t}, \beta 5, \beta 5 \mathrm{i}$, and $\alpha 6 \mathrm{Abs}$. Tissue distribution of $\beta 5$ and $\beta 5 \mathrm{i}$ was similar in WT and Tg mice. B: Incorporation of $\beta 5 \mathrm{t}$ into $20 \mathrm{~S}$ proteasomes in $\mathrm{Tg}$ mice. Extracts from thymus, spleen, and skeletal muscle were immunoprecipitated with $\mathrm{Ab}$ for $\alpha 6$, followed by immunoblotting with the same four Abs. $\beta 5$ t was incorporated into $20 \mathrm{~S}$ proteasomes in the tissues of $\mathrm{Tg}$ mice. $\beta 5$-containing $20 \mathrm{~S}$ proteasomes virtually disappeared from the muscle of $\mathrm{Tg}$ mice. In the spleen of Tg mice, $\beta 5 \mathrm{i}$-containing $20 \mathrm{~S}$ proteasomes were detected along with $\beta 5$ t-containing $20 \mathrm{~S}$ proteasomes. C: Proteasomal activities in Tg mice. FCs and hepatocytes isolated from WT or Tg mice were subjected to cell-based luminescence assays using specific luminogenic proteasome substrates: Suc-LLVY-aminoluciferin for chymotrypsin-like activity, Z-LRR-aminoluciferin for trypsin-like activity, and Z-nLPnLD-aminoluciferin for caspase-like activity. The number of viable cells was measured by ATP assays using the luciferase reaction. Chymotrypsin-like activity was significantly decreased in FCs and hepatocytes from Tg mice. Data are expressed as the substrate luminescence/ATP luminescence ratio (mean $\pm \mathrm{SD}$ ). $n=4$ mice per group. ${ }^{*} P<0.05,{ }^{* * * *} P<0.001$ versus WT (Student's $t$-test). 
$\mathrm{mmol} / \mathrm{L}$ dithiothreitol, and centrifuged at $15,000 \times \mathrm{g}$ for 10 minutes. The levels of polyubiquitinated proteins were quantified by enzyme-linked immunosorbent assay according to the manufacturer's instructions (CycLex, Nagano, Japan). Levels of oxidized proteins were determined by sensitive procedures based on the reactivity of protein carbonyl with 2,4-dinitrophenylhydrazine, ${ }^{18,19}$ using an ELISA kit according to the manufacturer's instructions (BioCell, Auckland, New Zealand). Protein expression profiles were compared in muscles of WT and Tg mice with a Panorama antibody microarray XPRESS Profiler725 kit (Sigma-Aldrich), according to the manufacturer's instructions. Briefly, extracts from muscles of WT and $\mathrm{Tg}(1 \mathrm{mg} / \mathrm{mL})$ were labeled with Cy3 and Cy5 dyes, respectively, and the samples were applied simultaneously on the array. The expression profile of each sample (Cy3 and Cy5) was recorded individually and compared. The microarray slides were scanned on a GenePix 4000B scanner (Molecular Devices Japan, Tokyo, Japan). Data were normalized, and the ratio of Tg to WT mice was computed for each spot, to estimate the relative fold excess.

\section{Image Analyses}

The thickness of the dermal adipose and muscle layers, the amount of lipid accumulation in liver tissues and hepatocytes, and Western blotting data were subjected to image analysis using ImageJ software version 1.43u ( $\mathrm{NIH}$, Bethesda, MD).

\section{Statistical Analyses}

We performed statistical analyses for two unmatched groups with the unpaired, two-tailed Student's $t$-test. For the analysis of three or more unmatched groups, one-way analysis of variance with multiple comparisons or post hoc testing was performed. $P$ values of $<0.05$ were considered significant.

\section{Results}

\section{Decreased Body Weight and a Shortened Life Span in Tg Mice}

In Tg mice, $\beta 5 \mathrm{t}$ was expressed in all of the tissues tested and was incorporated into $20 \mathrm{~S}$ proteasomes, whereas in WT mice $\beta 5$ t was expressed only in the thymus (Figure 1, A and B). As expected, proteasomes in Tg mice showed decreased chymotrypsin-like activity, compared with those in WT mice (Figure 1C).

Tg mice were smaller in size and weighed significantly less than WT mice (Figure 2A). Furthermore, the survival rate was markedly reduced; more than half of the Tg mice died by 40 weeks (Figure 2B). Tg and WT mice showed similar food intake (Figure $2 \mathrm{C}$ ), and their blood total pro-
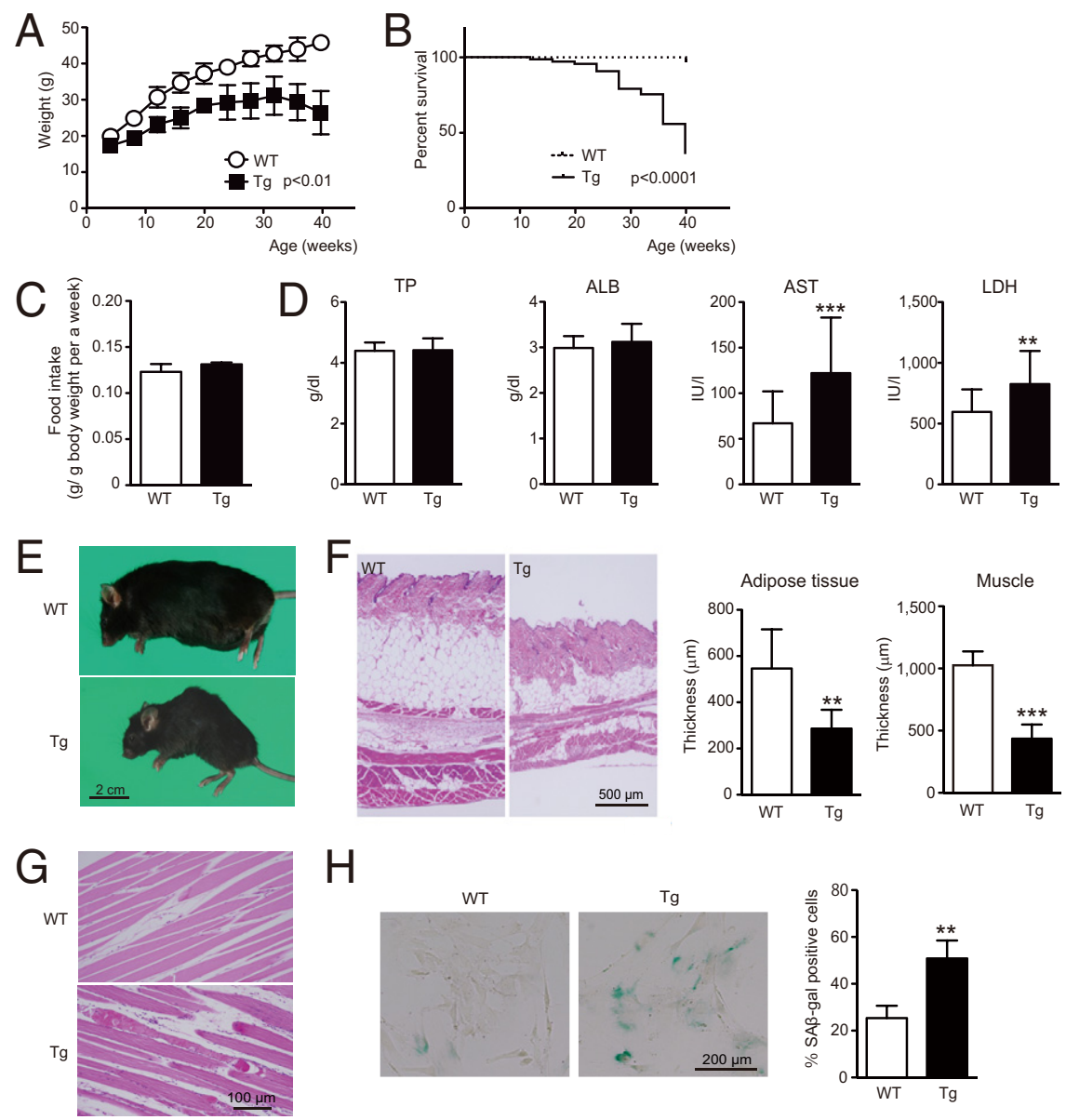

Figure 2. Decreased body weight and shortened life span in Tg mice. A: Body weight of male WT and Tg mice. $n=6$ to 10 mice per data point. B: Kaplan-Meier survival analysis of WT $(n=70)$ and $\operatorname{Tg}(n=75)$ cohort mice. C: Food intake was measured for 4 weeks in 16-week-old WT and Tg mice. $n=4$ mice per group. D: Plasma concentrations of total protein (TP), albumin (ALB), aspartate transaminase (AST), and lactate dehydrogenase (LDH) in WT and Tg mice. All mice were 4 to 24 weeks old. $n=24$ mice per group. E: Representative photographs of 24-week-old WT and Tg mice. Tg mice were smaller and appeared weak, compared with WT mice, and frequently exhibited lordokyphosis. Scale bar $=2 \mathrm{~cm}$. F: Subcutaneous adipose tissue and muscle mass in $\mathrm{Tg}$ mice (24 weeks of age) were decreased. The thickness was measured as described under Materials and Meth$o d s$. All mice were 24 weeks old. $n=6$ mice per group. Scale bar $=500 \mu \mathrm{m}$. G: Representative histological sections of skeletal muscle in WT and $\mathrm{Tg}$ mice. Atrophy and degeneration of muscle fibers appeared frequently in $\mathrm{Tg}$ mice older than 24 weeks of age. Scale bar $=100 \mu \mathrm{m}$. H: Cellular senescence in FCs from Tg mice. FCs from WT and Tg mice were stained with senescence-associated $\beta$-galactosidase solution. Positive cells were scored under light microscopy. $n=3$ mice per group. Scale bar $=200 \mu \mathrm{m}$. Data are expressed as means $\pm \mathrm{SD}$. ${ }^{* *} P<0.01,{ }^{* * * *} P<$ 0.001 versus WT (Student's $t$-test). 
tein and albumin levels were also similar (Figure 2D). Aspartate transaminase and lactate dehydrogenase levels were significantly increased in Tg mice (Figure 2D). Tg mice younger than 12 weeks of age displayed no apparent histological abnormality (see Supplemental Figure S1 at http://ajp.amjpathol.org). After 24 weeks of age, Tg mice gradually became weak, and died without any gross pathological changes such as cancer and cardiovascular diseases (data not shown). They frequently exhibited lordokyphosis (curvature of the spinal column) and a loss of subcutaneous adipose tissue and skeletal muscle mass (Figure 2, E and F). Microscopically, degeneration of skeletal muscle fibers was observed (Figure 2G). There were no phenotypic differences between male and female Tg mice (data not shown). Lordokyphosis and the loss of subcutaneous fat and skeletal muscle mass are prominent features of aging. In addition, primary FCs from Tg mice showed elevated levels of senescence-associated $\beta$-galactosidase, compared with those from age-matched WT mice (Figure $2 \mathrm{H}$ ). Thus, these results suggest that decreased chymotrypsin-like activity induces cellular senescence and leads to a shortened life span and age-related phenotypes.

\section{Accumulation of Polyubiquitinated and Oxidized Proteins in Tg Mice}

Proteasomes are responsible for the degradation of normal cellular proteins, as well as of abnormal proteins such as misfolded and oxidized proteins. We therefore investigated whether protein degradation by proteasomes is impaired in Tg mice. First, we investigated whether polyubiquitinated proteins are accumulated in the tissue lysates from muscles of $\mathrm{Tg}$ mice. Polyubiquitinated proteins were significantly increased in $\mathrm{Tg}$ mice (Figure 3A). Immunohistochemical analysis showed accumulation of Ub-conjugated substrates in both the nucleus and cytoplasm (Figure 3B). In addition, aggresomes were observed in FCs from $\mathrm{Tg}$ mice (see Supplemental Figure S2 at http://ajp.amjpathol.org). Next, using a protein array, we compared the expression of 725 proteins regulating cellular functions including apoptosis, cell cycle, signal transduction, and stress responses between $\mathrm{Tg}$ and WT mice. Expression of several cellular proteins was significantly altered in Tg mice (see Supplemental Table S1 at http://ajp.amjpathol.org). Notably, two cellular proteins regulating cellular senescence and survival showed altered expression: RNase $L$ was increased more than twofold, and $\mathrm{Bcl}-\mathrm{xL}$ was reduced more than twofold in $\mathrm{Tg}$ mice. These findings were confirmed by Western blot analysis (Figure 3C).

To examine whether the accumulated RNase $L$ proteins in $\mathrm{Tg}$ mice are polyubiquitinated, immunoprecipitates with anti-RNase $\mathrm{L} A \mathrm{Ab}$ were analyzed by Western blotting using anti-Ub Ab (Figure 3D). RNase L proteins in Tg mice were polyubiquitinated, suggesting that the accumulation of RNase $L$ proteins is due to decreased protein degradation resulting from decreased proteasomal activity. We tested the influence of proteasome inhibition on the level of RNase $L$ proteins by in vitro experiments using the proteasome inhibitor bortezomib.
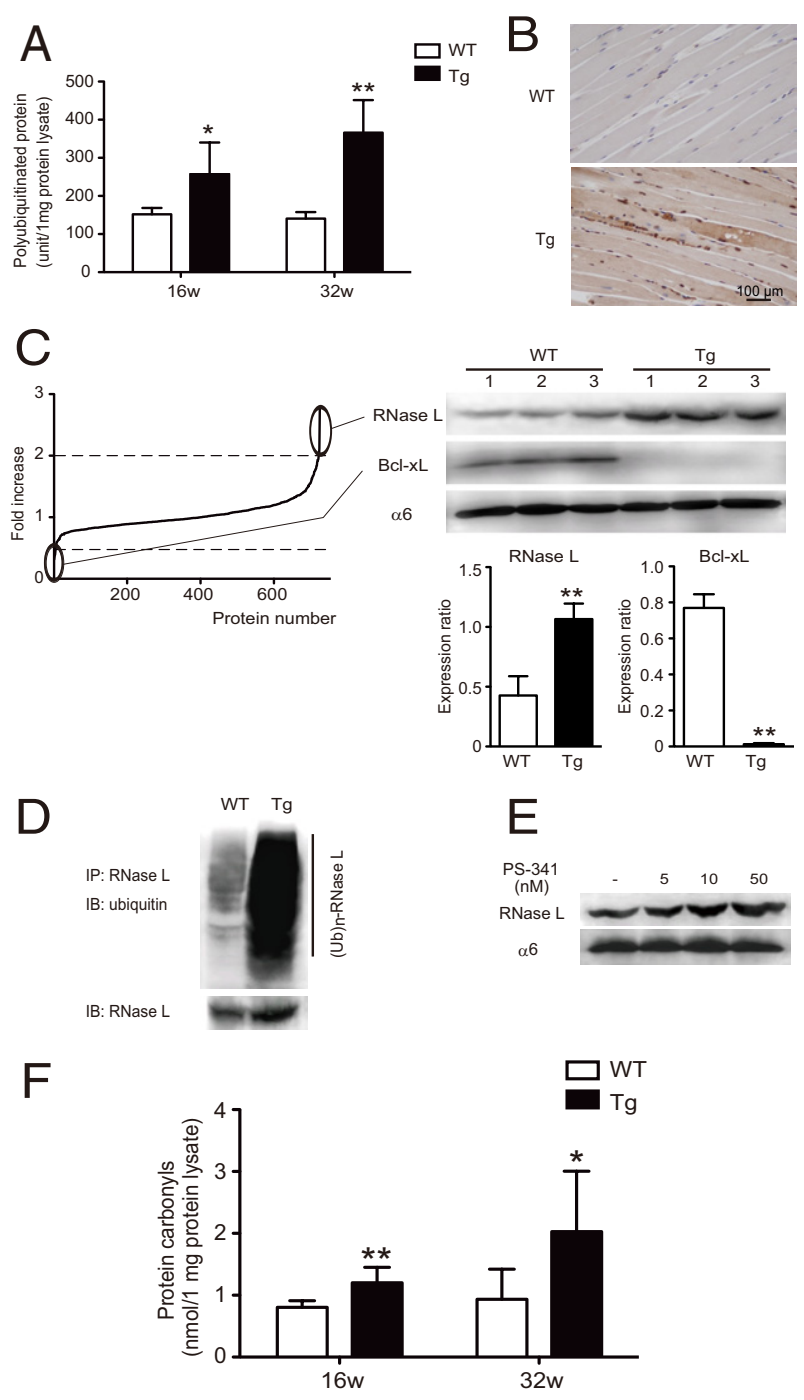

Figure 3. Accumulation of polyubiquitinated and oxidized proteins in $\mathrm{Tg}$ mice. A: Polyubiquitinated proteins in the muscles of WT and Tg mice at 16 and 32 weeks. $n=4$ mice per group. B: Immunohistochemical staining for Ub in the muscles of 24-week-old WT and Tg mice. Staining patterns are representative of four WT and four Tg mice. Scale bar $=100 \mu \mathrm{m}$. C: Altered expression of cellular proteins in Tg mice. Expression levels of 725 proteins extracted from skeletal muscles were compared between 24-week-old WT and Tg mice. Proteins whose expression levels differed more than twofold between WT and Tg mice are encircled. A comprehensive list of proteins with altered expression is given in Supplemental Table S1 (available at http://ajp.amipathol.org). Altered expression of RNase $\mathrm{L}$ and $\mathrm{Bcl}-\mathrm{xL}$ was validated by Western blot analysis. Lanes 1,2 , and 3 represent individual mice. The proteasome subunit $\alpha 6$ was used as an internal control. The expression ratio of RNase $\mathrm{L}$ and $\mathrm{Bcl}-\mathrm{xL}$ to $\alpha 6$ (right upper panel) was determined by image analysis of the Western blotting data (right lower panel). D: Polyubiquitinated RNase $\mathrm{L}$ is accumulated in Tg mice. Muscle tissue extracts from 24-week-old WT and Tg mice were immunoprecipitated (IP) with anti-RNase $\mathrm{L} \mathrm{Ab}$, followed by immunoblotting (IB) with anti-Ub $\mathrm{Ab}$. PolyUb-conjugated RNase L proteins typically appear as broad smears. E: RNase L accumulates in FCs treated with bortezomib (PS-341). FCs from WT mice were incubated in culture medium with $0,5,10$, or $50 \mathrm{nmol} / \mathrm{L}$ of bortezomib for 18 hours. After incubation, samples were subjected to Western blot analysis. F: Protein carbonyl concentrations in the muscles of WT and Tg mice at 16 and 32 weeks. $n=6$ mice per group. Data are expressed as means $\pm \mathrm{SD} .{ }^{*} P<0.05,{ }^{* *} P<0.01$ versus WT (Student's $t$-test).

The degradation of RNase $L$ was inhibited by bortezomib in a concentration-dependent manner (Figure $3 \mathrm{E}$ ). Because proteasomal activity decreases with age, ${ }^{8-11}$ we compared expression of $\mathrm{Bcl}-\mathrm{xL}$ and RNase $\mathrm{L}$ between young ( 16 weeks old) and aged (92 weeks old) WT mice. 

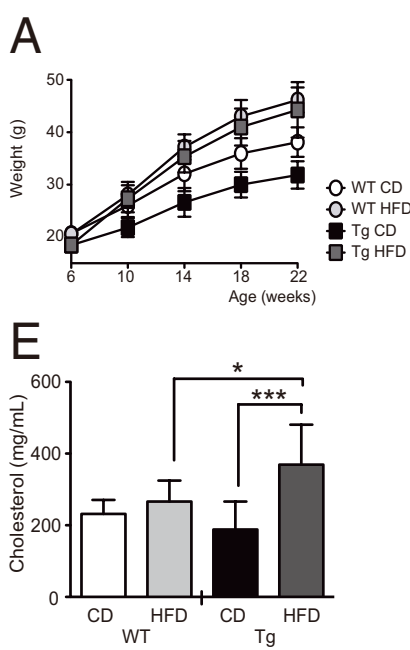

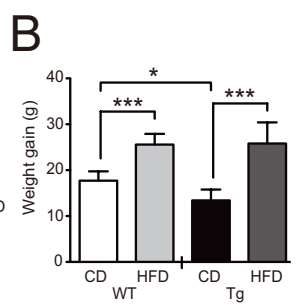

$\mathrm{F}$

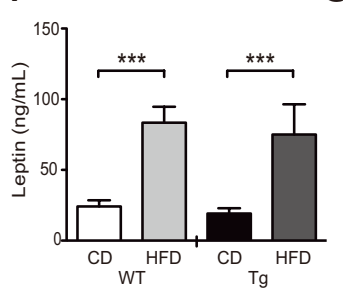

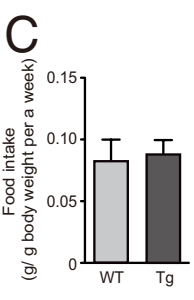

G

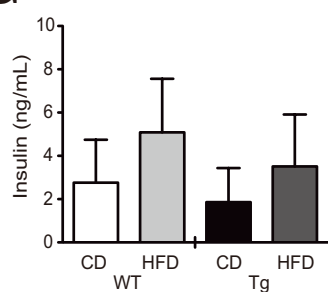

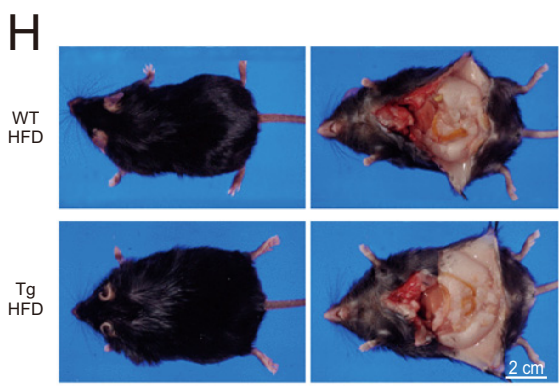

(1) Obesity in HFD-fed Tg mice. A: Body weight of CD- or HFD-fed WT and Tg mice. $n=10$ mice per data point. B: Weight gain in CD- or HFD-fed WT and Tg mice between the starting point ( 6 weeks old) and the endpoint ( 22 weeks old). $n=10$ mice per group. C: Food intake in HFD-fed WT and Tg mice was measured for 4 weeks in 16-week-old WT and Tg mice. $n=5$ mice per group. D: Weight gain after HFD feeding, calculated as the difference in body weight between CD-fed and HFD-fed mice. E: Total blood cholesterol concentrations in CD- or HFD-fed WT and Tg mice. F and G: Serum leptin (F) and insulin (G) concentrations in WT and Tg mice fed a CD or HFD. Data were collected at the endpoint (22 weeks old). $n=10$ mice per group. H: Representative photographs of HFD-fed WT and Tg mice. HFD-fed Tg mice were visibly more obese, and the deposition of subcutaneous and visceral fat was more pronounced, compared with HFD-fed WT mice. Scale bar $=2 \mathrm{~cm}$. Data are expressed as means $\pm \mathrm{SD} .{ }^{*} P<0.05,{ }^{* * * * *} P<0.001$ (one-way analysis of variance with multiple comparisons or post hoc testing).

Although expression of Bcl-xL was not significantly altered in aged WT mice, expression of RNase L was increased in aged WT mice, compared with young WT mice (see Supplemental Figure S3 at $h$ ttp://ajp.amjpathol.org).

We also measured protein carbonyl concentrations in the muscle as indices of oxidized proteins. In Tg mice, carbonylated proteins were increased (Figure 3F), indicating accumulation of oxidatively damaged proteins.

\section{Pronounced Obesity and Fat Accumulation in HFD-Fed Tg Mice}

Because proteasomal activity decreases with age, we hypothesized that a decline in proteasomal chymotrypsin-like activity in vivo might be involved in the development of age-related metabolic disorders. To test this hypothesis, we fed Tg mice a HFD and analyzed phenotypic alterations. Dietary fat is an important environmental factor promoting obesity, which in turn is critically involved in the development of many metabolic disorders. Administration of the HFD yielded more weight gain, compared with a CD, in both Tg and WT mice (Figure 4, $A$ and $B$ ). There was no difference in food intake between WT and Tg mice fed a HFD (Figure 4C). Of note, when Tg and WT mice were fed a HFD, the Tg mice gained more weight than the WT mice (Figure 4D), and total blood cholesterol levels increased more prominently in Tg than in WT mice (Figure 4E). Serum leptin was also elevated in both $\mathrm{Tg}$ and WT mice fed a HFD; however, the increase was not significantly different between $\mathrm{Tg}$ and WT mice (Figure 4F). There was no significant increase in the levels of serum insulin (Figure 4G). Fasting serum glucose levels were significantly increased in both WT and Tg mice fed a HFD, compared with CD $(P<0.05)$; however, there was no significant difference in glucose tolerance between
WT and Tg mice fed a HFD or between WT and Tg mice fed the CD (see Supplemental Figure S4 at http://ajp.amjpathol. org). Consistent with more weight gain, HFD feeding induced more prominent subcutaneous and visceral fat deposition in Tg than in WT mice (Figure $4 \mathrm{H}$ ).

Microscopically, marked hepatic steatosis was observed in both Tg and WT mice; however, the pattern of steatosis was different. Tg mice developed macrovesicular steatosis, whereas lipid droplets in WT mice were predominantly microvesicular (Figure 5A). Tg mice accumulated more lipids than WT mice, as measured by digital image analysis of Oil Red O-stained liver sections (Figure 5B). Although there was no statistical difference, triglyceride and cholesterol levels in the liver of HFD-fed Tg mice were increased, compared with HFD-fed WT mice (see Supplemental Figure S5 at $h$ ttp://ajp.amjpathol.org). Because lipid-droplet-associated proteins, such as perilipin, ADRP, and CIDE family proteins (CIDE A, CIDE B, and Fsp27), are known to associate with intracellular lipid droplets and to regulate their formation and metabolism, ${ }^{20,21}$ we examined their expression in the liver of $\mathrm{Tg}$ and WT mice. Among the proteins tested, expression of ADRP was significantly elevated in Tg mice (Figure 5C).

To examine whether decreased proteasomal activity promotes lipid accumulation, we performed in vitro experiments using primary hepatocytes. When hepatocytes isolated from WT mice were incubated in oleic acidcontaining medium (600 $\mu \mathrm{mol} / \mathrm{L})$, Oil Red O-stained lipid droplets were observed in hepatocytes (Figure 6A). When the cells were treated with bortezomib, the amount of lipid droplets was significantly increased and the size of lipid droplets in the hepatocytes was enlarged (Figure $6, A$ and $B$ ). Expression of ADRP was elevated when the cells were incubated with oleic acid and bortezomib (Figure $6 \mathrm{C}$ ), with ADRP distributed around lipid droplets in 

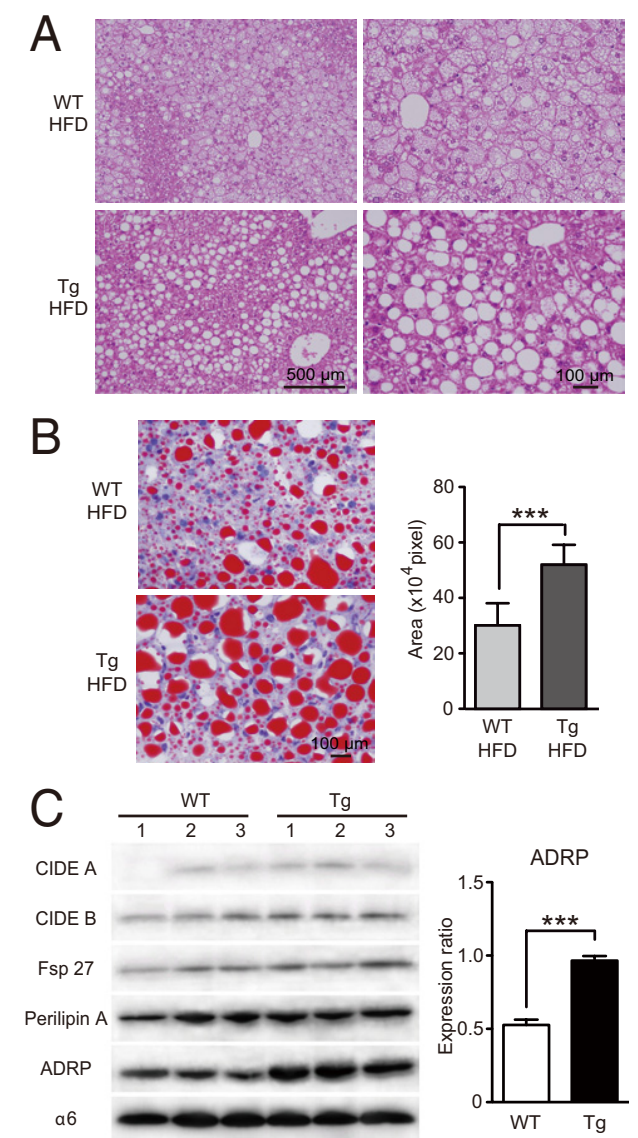

Figure 5. Steatosis and fat accumulation in HFD-fed Tg mice. A: H\&E staining of liver sections from HFD-fed WT and Tg mice. Scale bars: $500 \mu \mathrm{m}$ (left); 100 $\mu \mathrm{m}$ (right). B: Oil Red O staining of liver sections from HFD-fed WT and Tg mice. The amount of accumulated lipid in the liver of HFD-fed WT and Tg mice was measured by digital image analysis. $n=10$ mice per group. C: Expression of lipid-droplet-associated proteins in Tg mice. Extracts from livers of 24-weekold WT and Tg mice were immunoblotted with six different Abs. Lanes 1, 2, and 3 represent individual mice. $n=3$ mice per group. ADRP, adipose differentiation-related protein; CIDE A and B, cell death-inducing DNA fragmentation factor-45-like effector protein A and B; Fsp 27, fat-specific protein 27. The expression ratio of ADRP to $\alpha 6$ was determined by image analysis of the Western blotting data. Data are expressed as means $\pm \mathrm{SD}$. ${ }^{* * * *} P<0.001$ (Student's $t$-test).

hepatocytes (see Supplemental Figure S6 at http:// ajp.amjpathol.org). These results indicate that decreased proteasomal activity promotes lipid accumulation, suggesting a possible mechanistic explanation for the obesity and hepatic steatosis observed in Tg mice.

\section{Pronounced Steatosis and Fat Accumulation in HFD-Fed Aged WT Mice}

Although the underlying molecular mechanism is poorly understood, proteasomal activities are known to decrease with age. ${ }^{8-11}$ We confirmed that hepatocytes from aged WT mice indeed had decreased proteasomal chymotrypsin-like activity, compared with those from young WT mice (Figure 7A). Tg mice showed significantly decreased chymotrypsin-like activity in comparison with age-matched WT mice; after 60 weeks of age, the chymotrypsin-like activities in WT mice were similar to those in Tg mice. To examine whether aged WT mice show metabolic abnormalities similar to those observed in $\mathrm{Tg}$ mice, 76-week-old WT mice were challenged with a HFD or CD. HFD feeding induced a significant increase in body weight and blood cholesterol levels, compared with CD feeding (Figure 7, B-D). Notably, a significant increase in blood cholesterol levels was observed in HFD-fed aged WT mice, but no significant increase was observed in HFDfed young WT mice (Figures 4E and 7D). When young and aged mice were fed a HFD, aged mice gained more weight than young mice (Figure 7E). Strikingly, like the Tg mice (Figure 5, A and B), the HFD-fed aged mice developed macrovesicular hepatic steatosis (Figure 7F) and accumulated more lipids, compared with HFD-fed young mice (Figure 7G). In addition, ADRP expression was increased in aged mice (Figure $7 \mathrm{H}$ ). Thus, Tg and aged WT mice exhibited similar metabolic abnormalities, and they both developed more pronounced obesity and hepatic steatosis than young WT mice when challenged with a HFD.

\section{Discussion}

An age-related decrease in proteasomal activity has been assumed to be involved in the aging process and the development of age-related pathology. ${ }^{8-10}$ To date, however, evidence supporting this assumption has been limited. In the present study, we generated for the first time an animal model in which proteasomal chymotrypsin-like activity is reduced. Mice with diminished chymotrypsin-like activity accumulated ubiquitinated as well as oxidized proteins (Figure 3), and their life span was shortened, with evidence of cellular senescence (Figure 2). They exhibited lordokyphosis and a loss of subcutaneous adipose tissue and skeletal muscle mass (Figure 2), both prominent features of normal aging. Importantly, these mice showed metabolic abnormalities typically seen in aged mice; they were susceptible to HFD-induced obesity and hepatic steatosis, and had elevated levels of

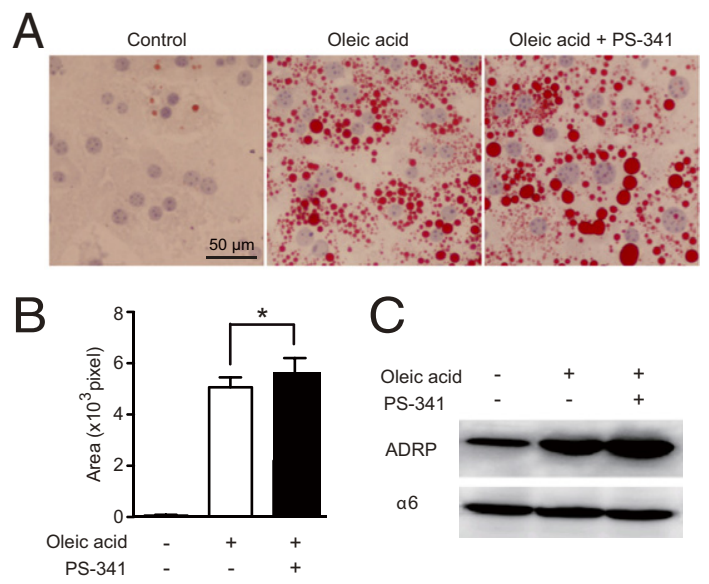

Figure 6. Decreased proteasomal chymotrypsin-like activity and lipid accumulation in hepatocytes. Hepatocytes from WT mice were incubated in oleic acid-containing medium $(600 \mu \mathrm{mol} / \mathrm{L})$ with or without $100 \mathrm{nmol} / \mathrm{L}$ bortezomib (PS-341) for 48 hours, and subjected to Oil Red O staining and Western blot analysis. A: Representative photomicrographs of Oil Red O staining of hepatocytes. Scale bar $=50 \mu \mathrm{m}$. B: Lipids in hepatocytes were measured by digital image analysis. Data were corrected by cell numbers. $n=10$ experiments/group. C: ADRP measured in hepatocytes treated with oleic acid and bortezomib. Data are expressed as means \pm SD. ${ }^{*} P<0.05$ (Student's $t$-test). 

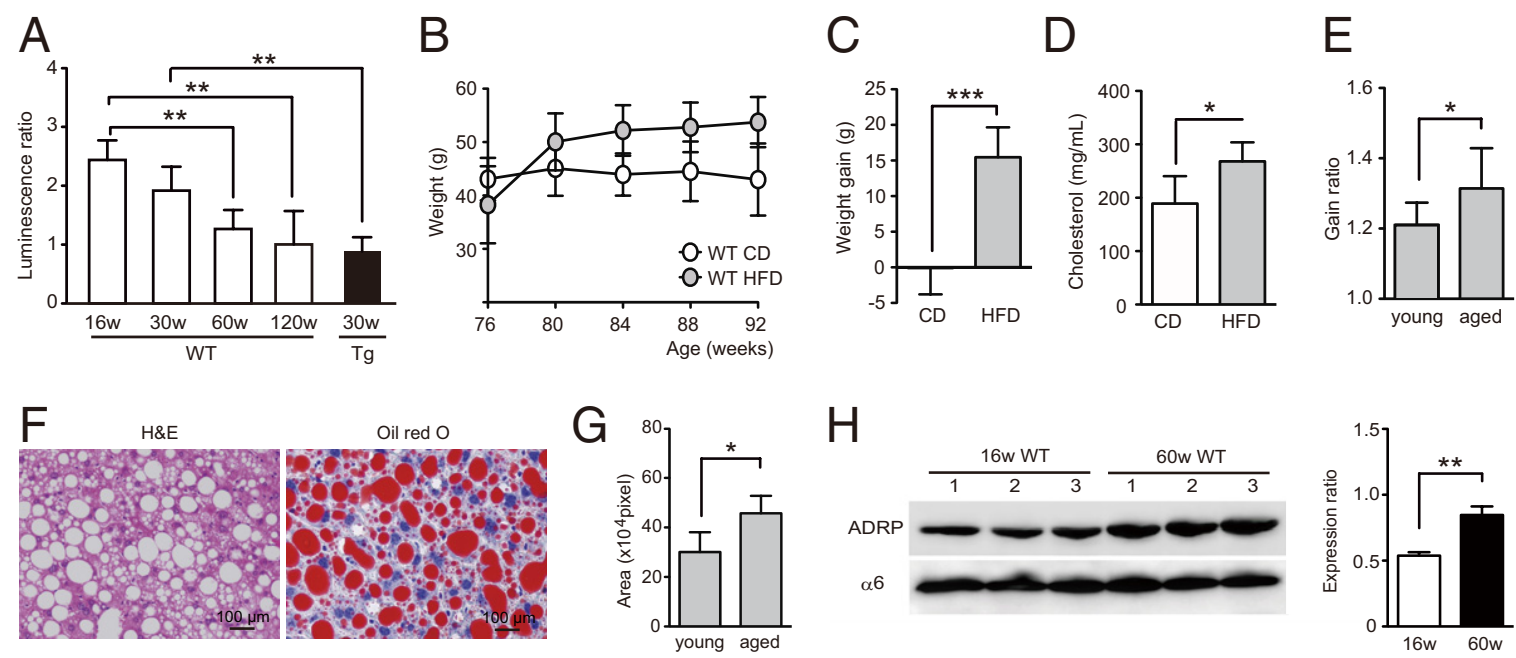

Figure 7. Steatosis and fat accumulation in HFD-fed aged WT mice. A: Proteasomal chymotrypsin-like activity in hepatocytes from WT and Tg mice. Isolated hepatocytes were subjected to luminescent assays using a specific luminogenic proteasome substrate, Suc-LLVY-aminoluciferin. The number of viable cells was measured by ATP assays using the luciferase reaction. Data are expressed as a Suc-LLVY luminescence/ATP luminescence ratio. $n=4,5$, or 6 mice per group. B: Body weight of aged CD- or HFD-fed WT mice. $n=4$ or 5 mice per data point. C: Weight gain in CD- or HFD-fed WT mice between starting point ( 76 weeks old) and the endpoint (92 weeks old). $n=4$ or 5 mice per group. D: Total blood cholesterol concentration (mg/mL) in CD- or HFD-fed WT mice. Data were measured at the endpoint ( 92 weeks old). $n=4$ or 5 mice per group. E: Weight gain in young (22 weeks old) and aged ( 92 weeks old) WT mice after HFD feeding. Fold increase in body weight was calculated by dividing the body weight of HFD-fed mice by that of CD-fed mice. F: H\&E and Oil Red O staining of liver sections from HFD-fed aged WT mice. Scale bars: $100 \mu \mathrm{m}$. G: Lipid accumulation in the liver of HFD-fed young ( 22 weeks old) and aged (92 weeks old) WT mice. $n=$ 4 to 10 mice per group. H: Expression of ADRP in aged WT mice. Liver extracts from young (16 weeks old) and aged (60 weeks old) WT mice were immunoblotted with ADRP Ab (left panel). Lanes 1, 2, and 3 represent individual mice. The expression ratio of ADRP to proteasome subunit $\alpha 6$ was calculated (right panel). $n=3$ mice per group. Data are expressed as means $\pm \mathrm{SD} .{ }^{*} P<0.05,{ }^{* *} P<0.01$, and ${ }^{* * *} P<0.001$ (Student's $t$-test).

ADRP (Figures 4, 5, and 7). Thus, our results suggest that decreased proteasomal activity accelerates the aging process and promotes the development of age-related pathology.

Ub-conjugated substrates are known to accumulate in most aged tissues because of decreased removal by proteasomes. $^{22,23}$ However, little is known about the identity of specific substrate proteins that accumulate in the tissue of aged individuals. In the present study, using protein arrays we identified several proteins whose expression differed significantly between Tg and WT mice (Figure 3C and see Supplemental Table S1 at http:// ajp.amjpathol.org). RNase $\mathrm{L}$, whose expression was increased, is an effector molecule of the 2-5A system, a major enzymatic pathway regulated by interferons. ${ }^{24,25}$ RNase $L$ expression is increased in an age-dependent manner, and its ectopic expression induces a senescent morphology and accelerated replicative senescence in cultured cells. ${ }^{25,26}$ Furthermore, RNase L knockout mice survive longer than RNase L WT mice. ${ }^{27}$ On the other hand, $\mathrm{Bcl}-\mathrm{xL}$, whose expression was decreased in $\mathrm{Tg}$ mice, is an antiapoptotic protein that plays a central role in cell survival; it inhibits senescence induction in cultured cells by preventing generation of reactive oxygen species. ${ }^{28}$ Thus, elevated expression of RNase $L$ and decreased expression of $\mathrm{BCl}-\mathrm{xL}$ may be involved in the development of age-related phenotypes in Tg mice.

As in Tg mice, expression of RNase $L$ was elevated in aged WT mice. In contrast, Bcl-xL expression was not significantly altered in aged mice (see Supplemental Figure S3 at http://ajp.amjpathol.org) whereas it was reduced in Tg mice. This discordance may be explained if reduction in proteasomal activity is more persistent and profound in Tg than in aged WT mice. Alternatively, substrate specificity of the proteasome may be altered by the incorporation of the $\beta 5 \mathrm{t}$ subunit. Bcl- $\mathrm{xL}$ is transcriptionally regulated by $\mathrm{NF}-\kappa \mathrm{B}$, and activation and nuclear translocation of $\mathrm{NF}-\kappa \mathrm{B}$ are dependent on the degradation of inhibitory- $\kappa \mathrm{B}\left(\mathrm{I}_{\kappa \mathrm{B}}\right)$ by proteasomes. ${ }^{29}$ Incorporation of $\beta 5 t$ may, for example, alter the substrate specificity of the proteasome so that $\left.\right|_{\kappa} \mathrm{B}$ becomes more resistant to proteasomal degradation. Because altered substrate specificity of the proteasome should affect degradation of many other proteins, it may make a large contribution to the phenotypes of Tg mice, along with decreased chymotrypsin-like activity. In addition to RNase $\mathrm{L}$ and $\mathrm{Bcl}-\mathrm{xL}$, we identified several proteins that were differentially expressed in Tg and WT mice, but with no known functions in cellular senescence (see Supplemental Table S1 at http://ajp.amjpathol.org). Further studies of these proteins may provide new insights into the regulation of cellular senescence.

In addition to Ub-conjugated proteins, Tg mice accumulated oxidized proteins (Figure 3F). Oxidatively damaged proteins, which are normally removed by proteasomes, ${ }^{30,31}$ are widely believed to contribute to the aging process. ${ }^{31,32}$ Thus, the accumulation of oxidatively damaged proteins presumably constitutes an additional factor contributing to the age-related phenotype in Tg mice.

Many human diseases are age-related, and aging is known to increase the prevalence of metabolic disorders. Metabolic syndrome occurs more frequently in the elderly, and intake of high dietary fat has been shown to induce more fat accumulation in aged than in young individuals. ${ }^{33-36}$ It has been assumed that altered physical activity and systemic metabolism, such as a reduction in systemic energy expenditure, are major risk factors for the development of metabolic disorders such as 
obesity, hyperlipidemia, and liver steatosis. With the present study, we have provided in vivo and in vitro evidence linking decreased proteasomal activity and lipid accumulation. Our data suggest that, in addition to the aforementioned risk factors, an age-related decline in proteasomal activity is involved in the development of age-related metabolic disorders. Remarkably, HFD-fed aged WT mice developed more pronounced obesity and hepatic steatosis than young WT mice (Figure 7), suggesting that the metabolic abnormalities in Tg mice mimic those in aged mice. Specifically, ADRP expression was increased in the liver of both Tg and aged WT mice. ADRP is a constitutively expressed, lipid-droplet-associated protein that is rapidly degraded by proteasomes in the absence of lipids. ${ }^{37-39}$ Its abundance is directly proportional to the amount of intracellular lipids, and its expression is increased in human diseases involving fat accumulation, such as hepatic steatosis. ${ }^{40,41}$ Thus, upregulated expression of ADRP may in part account for the pronounced HFD-induced obesity and hepatic steatosis observed in Tg and aged WT mice.

In conclusion, the newly developed Tg model described here enabled us to address the in vivo significance of proteasomal chymotrypsin-like activity for the first time. Our results suggest that an age-related decline in proteasomal activity has an important role in the pathogenesis of age-related metabolic disorders and possibly in age-related disorders in general. Future studies are needed to address the molecular mechanisms underlying an age-related decrease in proteasomal activity and to identify specific substrate proteins, the altered expression of which is involved in the development of agerelated diseases. Such studies may provide a new approach to the prevention and treatment of age-related diseases.

\section{Acknowledgments}

We thank Chisato Sudo, Saori Konno, Yuki Matsui, and Dr. Mitsufumi Nishio (Hokkaido University Graduate School of Medicine) for technical support and the staff of the Institute for Animal Experimentation (Hokkaido University Graduate School of Medicine) for mouse maintenance.

\section{References}

1. Ciechanover A: Proteolysis: from the lysosome to ubiquitin and the proteasome. Nat Rev Mol Cell Biol 2005, 6:79-87

2. Coux O, Tanaka K, Goldberg AL: Structure and functions of the $20 \mathrm{~S}$ and $26 S$ proteasomes. Annu Rev Biochem 1996, 65:801-847

3. Kloetzel PM: The proteasome and MHC class I antigen processing. Biochim Biophys Acta 2004, 1695:225-233

4. Baumeister W, Walz J, Zühl F, Seemüller E: The proteasome: paradigm of a self-compartmentalizing protease. Cell 1998, 92:367-380

5. Kisselev AF, Callard A, Goldberg AL: Importance of the different proteolytic sites of the proteasome and the efficacy of inhibitors varies with the protein substrate. J Biol Chem 2006, 281:8582-8590

6. Ludwig H, Khayat D, Giaccone G, Facon T: Proteasome inhibition and its clinical prospects in the treatment of hematologic and solid malignancies. Cancer 2005, 104:1794-1807

7. Voorhees PM, Dees EC, O'Neil B, Orlowski RZ: The proteasome as a target for cancer therapy. Clin Cancer Res 2003, 9:6316-6325
8. Gaczynska M, Osmulski PA, Ward WF: Caretaker or undertaker? The role of the proteasome in aging. Mech Ageing Dev 2001, 122:235254

9. Chondrogianni N, Gonos ES: Proteasome dysfunction in mammalian aging: steps and factors involved. Exp Gerontol 2005, 40:931-938

10. Dahlmann B: Role of proteasomes in disease. BMC Biochem 2007, 8 Suppl 1:S3

11. Tonoki A, Kuranaga E, Tomioka T, Hamazaki J, Murata S, Tanaka K, Miura M: Genetic evidence linking age-dependent attenuation of the $26 S$ proteasome with the aging process. Mol Cell Biol 2009, 29:10951106

12. Sasaki K, Hamazaki J, Koike M, Hirano Y, Komatsu M, Uchiyama Y, Tanaka K, Murata S: PAC1 gene knockout reveals an essential role of chaperone-mediated $20 \mathrm{~S}$ proteasome biogenesis and latent $20 \mathrm{~S}$ proteasomes in cellular homeostasis. Mol Cell Biol 2010, 30:3864-3874

13. Murata S, Sasaki K, Kishimoto T, Niwa S, Hayashi H, Takahama Y, Tanaka K: Regulation of CD8+ T cell development by thymus-specific proteasomes. Science 2007, 316:1349-1353

14. Tomaru U, Ishizu A, Murata S, Miyatake Y, Suzuki S, Takahashi S, Kazamaki T, Ohara J, Baba T, Iwasaki S, Fugo K, Otsuka N, Tanaka $\mathrm{K}$, Kasahara M: Exclusive expression of proteasome subunit $\beta 5 \mathrm{t}$ in the human thymic cortex. Blood 2009, 113:5186-5191

15. Nitta T, Murata S, Sasaki K, Fujii H, Ripen AM, Ishimaru N, Koyasu S, Tanaka K, Takahama Y: Thymoproteasome shapes immunocompetent repertoire of CD8+ T cells. Immunity 2010, 32:29-40

16. Murata S, Yashiroda H, Tanaka K: Molecular mechanisms of proteasome assembly. Nat Rev Mol Cell Biol 2009, 10:104-115

17. Niwa H, Yamamura K, Miyazaki J: Efficient selection for high-expression transfectants with a novel eukaryotic vector. Gene 1991, 108: 193-199

18. Levine RL, Garland D, Oliver CN, Amici A, Climent I, Lenz AG, Ahn BW, Shaltiel S, Stadtman ER: Determination of carbonyl content in oxidatively modified proteins. Methods Enzymol 1990, 186:464478

19. Levine RL, Williams JA, Stadtman ER, Shacter E: Carbonyl assays for determination of oxidatively modified proteins. Methods Enzymol 1994, 233:346-357

20. Gong J, Sun Z, Li P: CIDE proteins and metabolic disorders. Curr Opin Lipidol 2009, 20:121-126

21. Miura S, Gan JW, Brzostowski J, Parisi MJ, Schultz CJ, Londos C, Oliver B, Kimmel AR: Functional conservation for lipid storage droplet association among Perilipin, ADRP, and TIP47 (PAT)-related proteins in mammals, Drosophila, and Dictyostelium. J Biol Chem 2002, 277: 32253-32257

22. Carrard G, Bulteau AL, Petropoulos I, Friguet B: Impairment of proteasome structure and function in aging. Int J Biochem Cell Biol 2002, 34:1461-1474

23. Ciechanover A, Orian A, Schwartz AL: The ubiquitin-mediated proteolytic pathway: mode of action and clinical implications. J Cell Biochem Suppl 2000, 34:40-51

24. Zhou A, Hassel BA, Silverman RH: Expression cloning of 2-5A-dependent RNAase: a uniquely regulated mediator of interferon action. Cell 1993, 72:753-765

25. Bisbal C, Silverman RH: Diverse functions of RNase $L$ and implications in pathology. Biochimie 2007, 89:789-798

26. Floyd-Smith G, Denton JS: Age-dependent changes are observed in the levels of an enzyme mediator of interferon action: a (2'-5')A(n)-dependent endoribonuclease. Proc Soc Exp Biol Med 1988, 189:329-337

27. Andersen JB, Li XL, Judge CS, Zhou A, Jha BK, Shelby S, Zhou L, Silverman RH, Hassel BA: Role of 2-5A-dependent RNase- $L$ in senescence and longevity. Oncogene 2007, 26:3081-3088

28. Jung MS, Jin DH, Chae HD, Kang S, Kim SC, Bang YJ, Choi TS, Choi KS, Shin DY: Bcl-xL and E1B-19K proteins inhibit p53-induced irreversible growth arrest and senescence by preventing reactive oxygen speciesdependent p38 activation. J Biol Chem 2004, 279:17765-17771

29. Fennell DA, Chacko A, Mutti L: BCL-2 family regulation by the $20 \mathrm{~S}$ proteasome inhibitor bortezomib. Oncogene 2008, 27:1189-1197

30. Friguet B, Bulteau AL, Chondrogianni N, Conconi M, Petropoulos I: Protein degradation by the proteasome and its implications in aging. Ann N Y Acad Sci 2000, 908:143-154

31. Grimm S, Höhn A, Grune T: Oxidative protein damage and the proteasome. Amino Acids 2012, 42:23-38 
32. Johnson FB, Sinclair DA, Guarente L: Molecular biology of aging. Cell 1999, 96:291-302

33. Denys K, Cankurtaran M, Janssens W, Petrovic M: Metabolic syndrome in the elderly: an overview of the evidence. Acta Clin Belg 2009, 64:23-34

34. Zamboni M, Mazzali G, Zoico E, Harris TB, Meigs JB, Di Francesco V, Fantin F, Bissoli L, Bosello O: Health consequences of obesity in the elderly: a review of four unresolved questions. Int J Obes (Lond) 2005, 29:1011-1029

35. Nishikawa S, Yasoshima A, Doi K, Nakayama H, Uetsuka K: Involvement of sex, strain and age factors in high fat diet-induced obesity in C57BL/6J and BALB/cA mice. Exp Anim 2007, 56:263-272

36. Hoffler U, Hobbie K, Wilson R, Bai R, Rahman A, Malarkey D, Travlos G, Ghanayem BI: Diet-induced obesity is associated with hyperleptinemia, hyperinsulinemia, hepatic steatosis, and glomerulopathy in C57BI/6J mice. Endocrine 2009, 36:311-325

37. Brasaemle DL, Barber T, Wolins NE, Serrero G, Blanchette-Mackie EJ, Londos C: Adipose differentiation-related protein is an ubiqui- tously expressed lipid storage droplet-associated protein. J Lipid Res 1997, 38:2249-2263

38. Masuda Y, Itabe H, Odaki M, Hama K, Fujimoto Y, Mori M, Sasabe N, Aoki J, Arai H, Takano T: ADRP/adipophilin is degraded through the proteasome-dependent pathway during regression of lipid-storing cells. J Lipid Res 2006, 47:87-98

39. Xu G, Sztalryd C, Lu X, Tansey JT, Gan J, Dorward H, Kimmel AR, Londos C: Post-translational regulation of adipose differentiation-related protein by the ubiquitin/proteasome pathway. J Biol Chem 2005 , 280:42841-42847

40. Heid HW, Moll R, Schwetlick I, Rackwitz HR, Keenan TW: Adipophilin is a specific marker of lipid accumulation in diverse cell types and diseases. Cell Tissue Res 1998, 294:309-321

41. Motomura W, Inoue M, Ohtake T, Takahashi N, Nagamine M, Tanno $\mathrm{S}$, Kohgo $\mathrm{Y}$, Okumura $\mathrm{T}$ : Up-regulation of ADRP in fatty liver in human and liver steatosis in mice fed with high fat diet. Biochem Biophys Res Commun 2006, 340:1111-1118 